\title{
Investigations on Quasi-Steady Characteristics for an Airfoil Oscillating at Low Reduced Frequencies
}

\author{
Deepakkumar M. Sharma and Kamal Poddar \\ Department of Aerospace Engineering, Indian Institute of Technology Kanpur, Uttar Pradesh 208016, India \\ Correspondence should be addressed to Deepakkumar M. Sharma, dmsharma@iitk.ac.in
}

Received 29 August 2009; Revised 10 May 2010; Accepted 20 September 2010

Academic Editor: Hui Hu

Copyright ( $) 2010$ D. M. Sharma and K. Poddar. This is an open access article distributed under the Creative Commons Attribution License, which permits unrestricted use, distribution, and reproduction in any medium, provided the original work is properly cited.

\begin{abstract}
Wind tunnel experiments were conducted on NACA-0015 airfoil model to investigate the effect of the reduced frequency $(\kappa=0.0001$ to 0.5$)$ and that Reynolds number $(\mathrm{Re}=0.2 E 06$ to $0.7 E 06)$ on the aerodynamic characteristics and hysteresis behavior associated with the oscillating motion of the airfoil. Pressure measurements were conducted on the midspan of the airfoil for quantitative results. Although dynamic stall study has been conducted to greater extent of reduced frequencies, for current work, emphasis is made on investigating the low reduced frequency regimes up to which the steady conditions prevail under dynamic mode of operation. In present investigations of oscillating sinusoidal $\alpha=10^{\circ}+15^{\circ} \sin (\omega t)$ motion, the airfoil was allowed to execute oscillating motion from static to almost steady (quasi-steady) conditions with the incremental increase in the reduced frequency. The limiting conditions of $\kappa$ and Re for quasi-steady state are carefully extracted and analyzed. Static and quasi-steady conditions are critically assessed in context of the existence of laminar separation bubble within the opted Re regime. The flow separation was found to be trailing edge flow separation with existence of LSB upstream towards the leading edge with no evidence of unsteady flow reversals. Normal force defect and pitch damping factor are estimated for varied range of Re and $\kappa$ within the quasi-steady conditions. Re effect is dominant at steady and quasi-steady regimes while the effect of reduced frequency is negligible in this domain. Minor hysteresis effect associated with the stalling and reattachment point is due to existence of weak stall flutter which is further restrained by the oscillatory motion of the airfoil. Laminar separation bubble traced during steady condition is conserved in quasi-steady domain as well.
\end{abstract}

\section{Introduction}

Dynamic stall phenomena are the result of airfoil and wing undergoing ramp or oscillatory motion and having a maximum angle of attack greater than the static stall angle. These unsteady flows are characterized by massive separation and formation of large-scale vortical structures. Numerous experimental and computational investigations [1-16] have shown that the unsteady flow can be separating or reattaching over a large portion of the top surface of the airfoil. The predominant feature of dynamic stall is the formation, shedding, and convection over the upper surface of the airfoil of an energetic vortex-like disturbance from the leading edge of the airfoil. It induces a nonlinearity fluctuating pressure field and produces transient variations in forces and moments that are fundamentally different from their steady state counterparts.
As shown in Figure 1 [17], in steady flow, separation of a boundary layer is said to occur where the boundary-layer flow velocity near the surface is reduced to zero and the flow detaches from the wall to form a wake that enclosed a reversed-flow region. The separation and flow reversal points are coincident, and the external stream is always distorted. In unsteady flow as shown in Figure 2 [17], the locations of the point of flow reversal and the point of flow separation are two distinctly different points. Goldstein [18] in his theoretical studies of laminar flows has shown that the boundary layer equations have a singularity at the point of flow reversal in steady flow. But in unsteady flow as shown by Sears and Telionis [19], the location of singularity associated with separation is distinctly different from the location of flow reversal. Thus, there exists an unsteady boundary layer that has a region of reversed flow near the surface, while not showing any strong variations in the boundary 


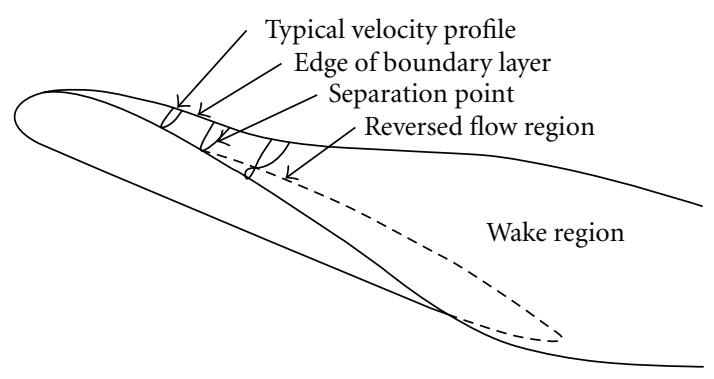

Figure 1: Steady separation on an airfoil [17].

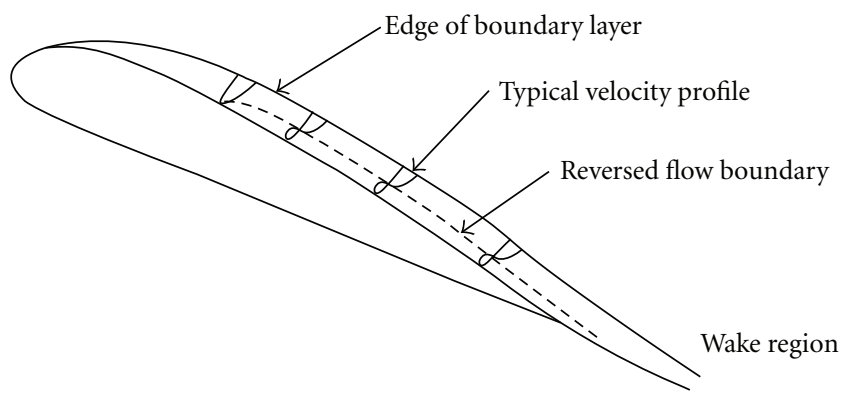

FIGURE 2: unsteady flow reversal on an oscillating airfoil [17].

layer displacement or momentum thickness and little or no distortion of the external stream. This distinction between flow reversal and separation has also been demonstrated by Nash et al. [20] for unsteady turbulent boundary layer flows.

Two quantities which summarize the degree of hysteresis associated with dynamic stall are given by the normal force defect $(\xi)$ and the pitch damping factor $(\zeta)$. $\xi$ is obtained from the close line integral around the cycle of the normal force, and $\zeta$ is obtained from the closed line integral of the pitching moment; in both cases the variable of integration is angle of attack $\alpha$ [21]:

$$
\begin{gathered}
\xi=\frac{\oint C_{n} d \alpha}{\int_{-\pi / 2}^{\pi / 2} C_{n} d \alpha}, \quad \zeta=-\oint C_{m} d \alpha / 4 \alpha_{1}^{2}, \\
C_{n}=\frac{1}{c}\left[\int_{0}^{c}\left(C_{p l}-C_{p u}\right) d x\right] \\
C_{a}=\frac{1}{c}\left[\int_{0}^{c}\left(C_{p u} \frac{d y_{u}}{d x}-C_{p l} \frac{d y_{l}}{d x}\right) d x\right] \\
\therefore C_{m L E}=\frac{1}{c^{2}}\left[\int_{0}^{c}\left(C_{p l}-C_{p u}\right) x d x\right] \\
\therefore C_{m}=C_{m L E}+\frac{C_{n}}{4} \quad(\text { moment about quarter-chord). }
\end{gathered}
$$

The normal force defect represents the loss in load carrying ability arising from the fact that the normal force experienced during the pitch-down is usually less than that during the pitch up. If there is no hysteresis, both portions of the cycle trace out an identical curve, and the defect is zero. The numerical value assigned to this hysteresis is therefore based on that percentage of the integral contributed during

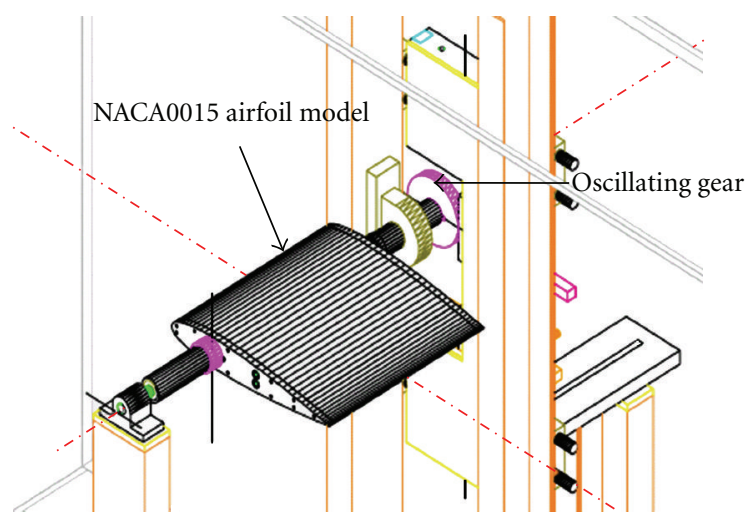

FIgURE 3: Experimental Set-up.

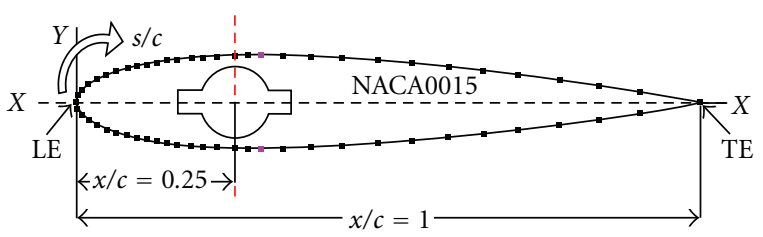

FIgURe 4: Pressure port locations of NACA0015.

the upstroke which is lost during the downstroke. The pitch damping term, which is more frequently reported, has a different interpretation. Since the pitching moment acts in the direction of motion of the airfoil, the closed-line integral of the pitching moment with respect to $\alpha$ provides a measure of the net accumulation of energy from the flow. Hence, if the normal force and pitching moment trace out clockwise patterns when viewed as a function of $\alpha$, then the normalforce defect will be positive, and the pitch damping will be negative. Within quasi-steady conditions, values of $\xi$ and $\zeta$ are quantified at varied Re and $\kappa . C_{n}-C_{m}$ correlation plots are formed by computing following nondimensional parameters [17]:

$$
\chi=\frac{C_{n}-C_{n_{0}}}{C_{n_{\max }}-C_{n_{0}}}, \quad \gamma=\frac{C_{m}-C_{m_{0}}}{C_{m_{\max }}-C_{m_{0}}},
$$

where $C_{n_{0}}$ and $C_{m_{0}}$ are taken at $\alpha=-4^{\circ}$. This nondimensionalization emphasizes the chronology of the significant events occurring during fully developed dynamic stall. It signifies the strength of hysteresis and identifies traces of weak or strong vortices or fluctuations generated during the oscillation cycle for a wide range of $\kappa$ and Re, thus forms a valuable technique for correlating dynamic stall test results.

The objective of current work is to determine and investigate the conditions for quasi-steady state. It could be defined as an unsteady state up to which steady flow conditions and static stall characteristics prevail. Thus, for given quasi-steady flow conditions, although the flow is unsteady, the boundary layer separation should have a unique singularity with no flow reversals, and moment stall and lift stall are presumably the same point. Also, as stated by Ericsson [22], both the frequency-induced camber ( $q$-effect) 


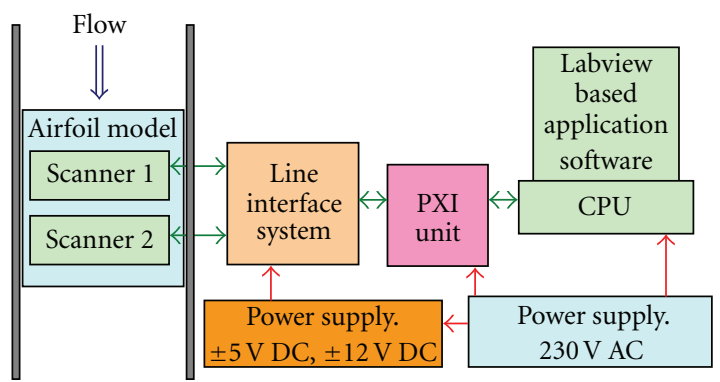

Figure 5: Measurement chain for surface pressure data acquisition.

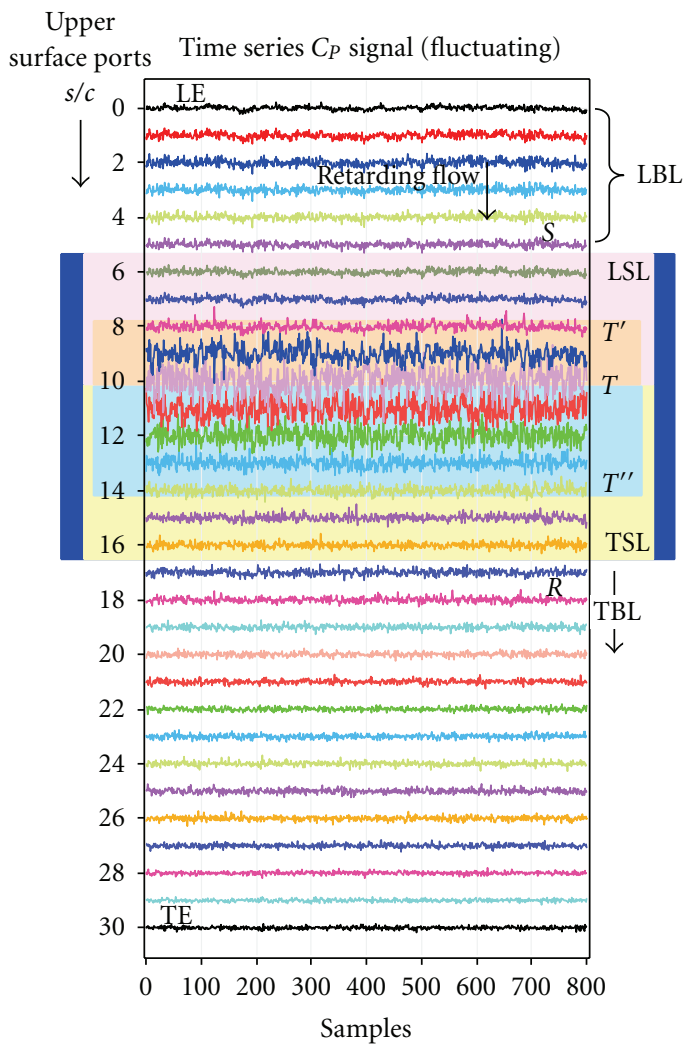

Figure 6: Time series $C_{P}$ signals (fluctuating) at $\mathrm{Re}=0.2 E 06$ and $\alpha=9.5^{\circ}$.

and the frequency-induced plunging ( $\dot{\alpha}$-effect) tend to zero. The set of very low-ordered reduced frequencies at varied Re are extracted and critically assessed to limit the quasi-steady conditions.

\section{Experimental Methodology}

Figure 3 shows the designed experimental set-up which generates simple harmonic oscillating motion for the given airfoil. The preferred mechanism (Scotch-yoke) is robust and capable of functioning at predefined inertial and fluid dynamic loads by giving due considerations to all its operating parameters. The airfoil model shaft passing through quarterchord is mounted on an oscillating gear performing sinusoidal motion. Limiting to the cross-sectional size

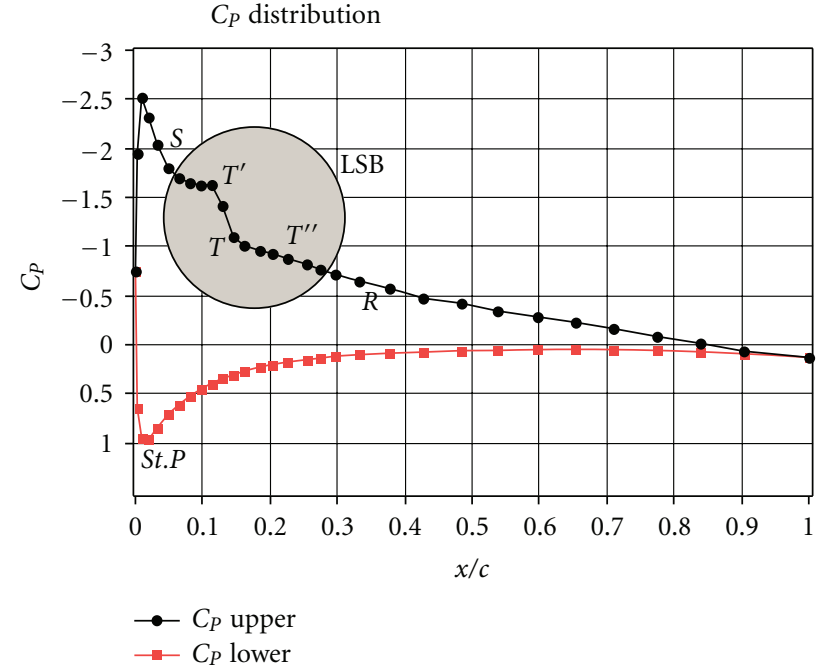

(a)

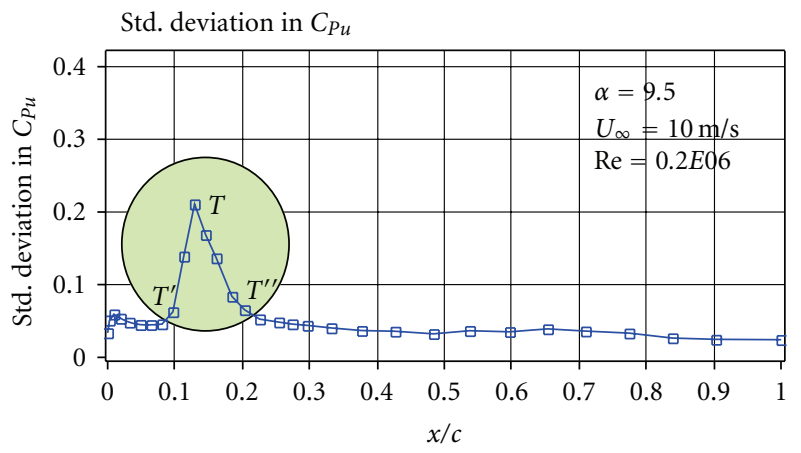

(b)

FIgURE 7: $C_{P}$ distribution and its standard deviation at $\mathrm{Re}=0.2 E 06$ and $\alpha=9.5^{\circ}$.

for one of the existing wind tunnel test section $(1.22 \mathrm{~m}$ $\times 0.305 \mathrm{~m}$ ), available at low speed aerodynamics lab of aerospace engineering department at IIT-Kanpur, the chord length of the selected NACA0015 airfoil is kept $0.31 \mathrm{~m}$ spanning the tunnel width, keeping the geometric blockage up to $10 \%$ at maximum angle of attack of $25^{\circ}$. Free stream velocity $\left(U_{\infty}\right)$ ranges from 10 to $35 \mathrm{~m} / \mathrm{s}$. Accordingly, Re ranges from $0.2 E 06$ to $0.7 E 06$ with $\kappa$ ranging from 0.001 to 0.5 for oscillation frequency $\left(f_{o}\right)$ varying from 0.01 to $5.4 \mathrm{~Hz}$. The free-stream turbulence level in the test section is within $0.15 \%$.

2 ESP Scanners (each having 32 ports) are used to measure the surface pressure all along the 60 ports opted on the mid-span of the airfoil model along the chord as shown in Figure 4. The multiplexing frequency of each scanner is $20,000 \mathrm{~Hz}$, and the accuracy is of $0.05 \%$ for the full range of $\pm 5000 \mathrm{~Pa}$. Figure 5 indicates the detailed measurement and instrumentation chain used for the surface pressure measurements. For both static and dynamic analysis, the time series pressure data were acquired with a sampling 
TABLE 1: Nomenclature.

\begin{tabular}{lccc}
\hline Description & Symbol & Value & Unit \\
\hline Reduced frequency & $\kappa$ & $\omega c / 2 U_{\infty}$ & - \\
Chord length & $c$ & & $\mathrm{~m}$ \\
Circular frequency & $\omega$ & $2 \pi f_{o}$ & $\mathrm{rad} / \mathrm{s}$ \\
Oscillating frequency & $f_{o}$ & & $\mathrm{~Hz}$ \\
Free stream velocity & $U_{\infty}$ & & $\mathrm{m} / \mathrm{s}$ \\
Reynolds number & $\mathrm{Re}$ & $U_{\infty} c / v$ & - \\
Kinematic viscosity & $v$ & $1.5 E-5$ & $\mathrm{~m}^{2} / \mathrm{s}$ \\
Angle of incidence & $\alpha$ & & $\mathrm{deg}$. \\
Amplitude of oscillations & $\alpha_{1}$ & $15^{\circ}$ & $\mathrm{deg}$. \\
Time & $t$ & & $\mathrm{~s}$ \\
Normal force defect & $\xi$ & & - \\
Pitch damping factor & $\zeta$ & & - \\
Normal force coefficient & $C_{n}$ & & - \\
Axial force coefficient & $C_{a}$ & & - \\
Pitching Moment coefficient & $C_{m}$ & & - \\
Co-ordinates of airfoil profile & $x, y$ & & - \\
Coefficient of Pressure & $C_{P}$ & $2\left(p-p_{\infty}\right) / \rho U_{\infty}^{2}$ & - \\
Local static pressure & $p$ & & - \\
Free stream static pressure & $p_{\infty}$ & & - \\
Upper and lower surface & $u, l$ & & - \\
Leading and Trailing edge & $\mathrm{LE}, \mathrm{TE}$ & & - \\
Distance measured along the & $s$ & & - \\
surface & $\mathrm{LSE}$ & - \\
Coefficients of lift and pressure & $C_{l}, C_{d p}$ & & - \\
drag & $\mathrm{LBL}$ & & - \\
Laminar Boundary Layer & & & - \\
\hline Leading Edge Vortex & & & - \\
\hline
\end{tabular}

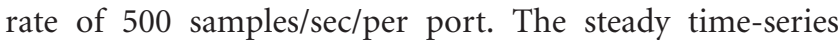
data taken for 10 seconds is averaged to get the mean $C_{P}$ distribution at a given angle of attack. The standard deviation in the time series $C_{P}$ signals is obtained which helps in tracing the evidence of LSB on the upper surface of the airfoil. The dynamic time series $C_{p}$ data is phaseaveraged for 50 cycles of oscillation. Uncertainty in the calculation of surface pressure coefficients $\left(C_{p}\right)$ is about $0.1 \%$.

\section{Results and Discussions}

\subsection{Steady State Analysis}

3.1.1. Tracing the Evidence of LSB. Figures 6 and 7 shows the sample procedure for identifying the occurrence of

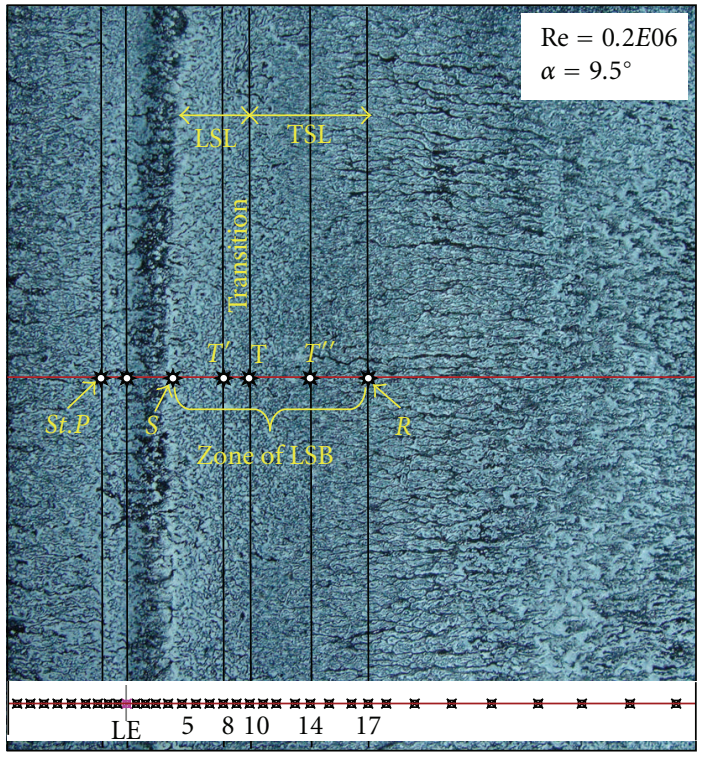

FIgURE 8: Surface flow visualization.

LSB in the flow phenomena by analyzing the fluctuating components of the acquired time-series $C_{P}$ signals at the mid-span of the airfoil. The mean and the Standard deviation of the $C_{P}$ signals are also shown in $C_{P}$ distribution and standard deviation plots, with respect to chord length $(x / c)$ for $\alpha=9.5^{\circ}$ and $\operatorname{Re}=0.2 E 06$. Attempt is made to correlate the larger magnitudes of disturbances observed in time series $C_{P}$ signals with $C_{P}$ distribution and standard deviation plots. The stagnation point (St.P) is at the lower surface (Port ID L3). From there along the surface, flow accelerates and initiates the growth of laminar boundary layer. Further it crosses LE and reaches $C_{P \min }$ up to which favorable pressure gradient holds (suction peak). The flow within the boundary layer then initiates to retard due to adverse pressure gradient and just aft of the suction peak after port ID U5, a sudden deviation is observed in $C_{P}$ plot with no significant change in pressure up to port ID U8. Port ID U5 is identified as Separation point $S$. Still the flow within the boundary layer is laminar as no deviation is observed in standard deviation plot. Separated laminar shear layer gets developed and extends up to port ID U10. At port ID U8, the standard deviation in $C_{P}$ signal just begins to grow and reaches the maximum at port ID 10. Ports ID U8 and U10 are identified as onset $T^{\prime}$ and extent $T$ of Transition. Laminar to Turbulent transition occurs at point $T$. As the turbulent shear layer grows, it sparks the turbulent mixing and entrainment of fluid from the free-stream until the pressure reaches the value of turbulent boundary layer on the surface. $T^{\prime \prime}$ indicates the point where entrainment processes ends (port ID U14) and the separated turbulent shear energizes to reattach on the surface at port ID U17 defined as reattachment point $R$. The entire chronology of the boundary layer events, viz, $S-T^{\prime}-T-T^{\prime \prime}-R$, defines the zone of LSB.

Figure 8 shows the surface oil-flow visualization image which is clearly distinct and identifies boundary layer 


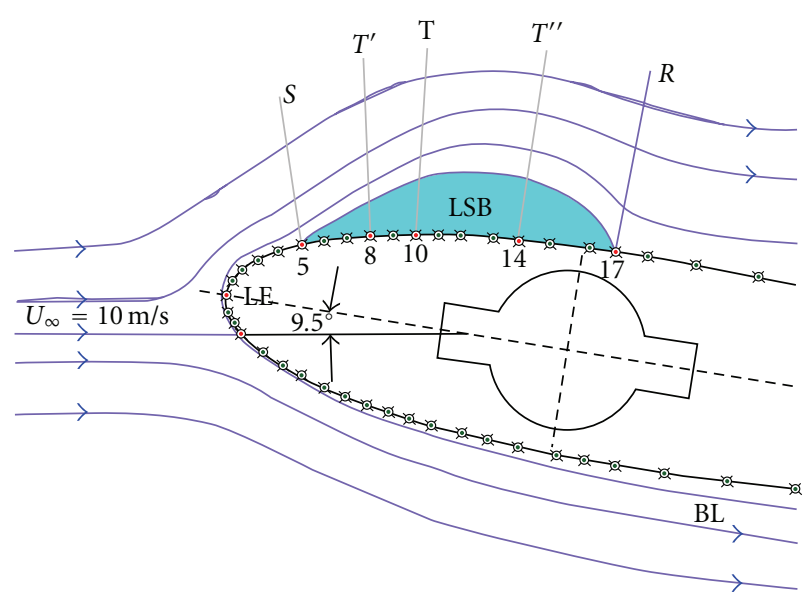

FIgURE 9: Schematic diagram of mean flow with LSB.

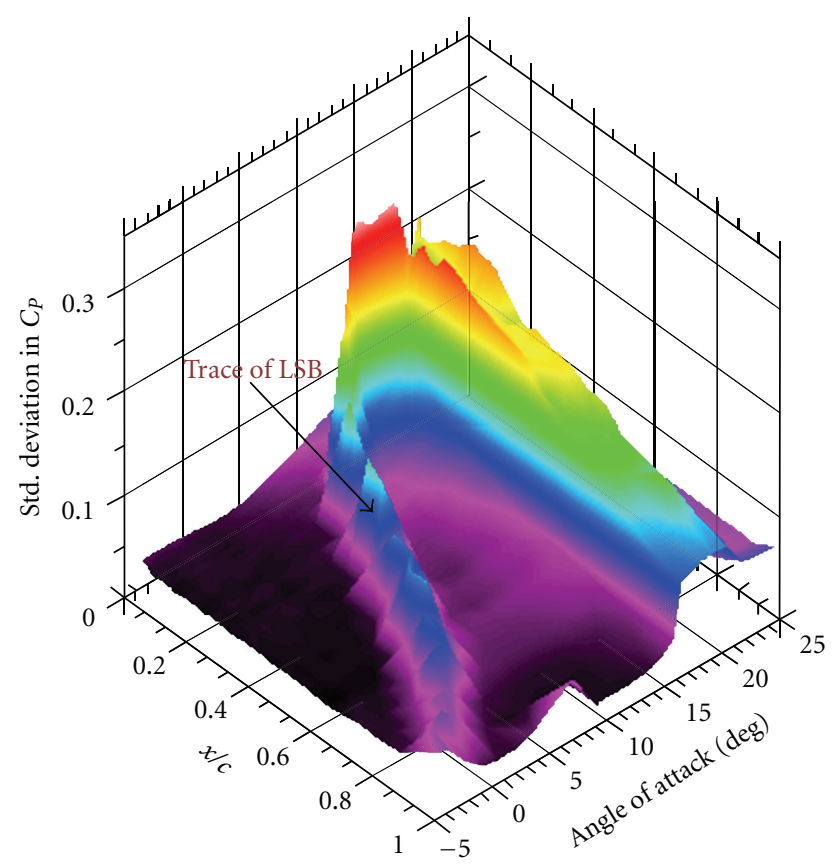

Figure 10: 3D plot of stander. deviation in $C_{P}$.

events. This technique has a great potential in identifying distinguished flow features on the surface. The skill lies in preparing the mixture of $\mathrm{TiO}_{2}+$ oleic acid + transformer-oil with definite proportions and tactfully applying it on the surface of the airfoil. The chronology of events determined between the separation and reattachment points quantifies the band identified as the zone of LSB. For the given case at $\operatorname{Re}=0.2 E 06$ and $\alpha=9.5^{\circ}$, the length of LSB along the surface $(s / c)$ comes out to be $=22.79 \%$ of the chord. The very existence of LSB classifies the flow separation to be a trailing edge turbulent boundary layer separation preceded by a laminar separation bubble. The results obtained through this technique are rightly compared with the boundary layer and laminar separation bubble parameters determined and validated with certain boundary layer approximation

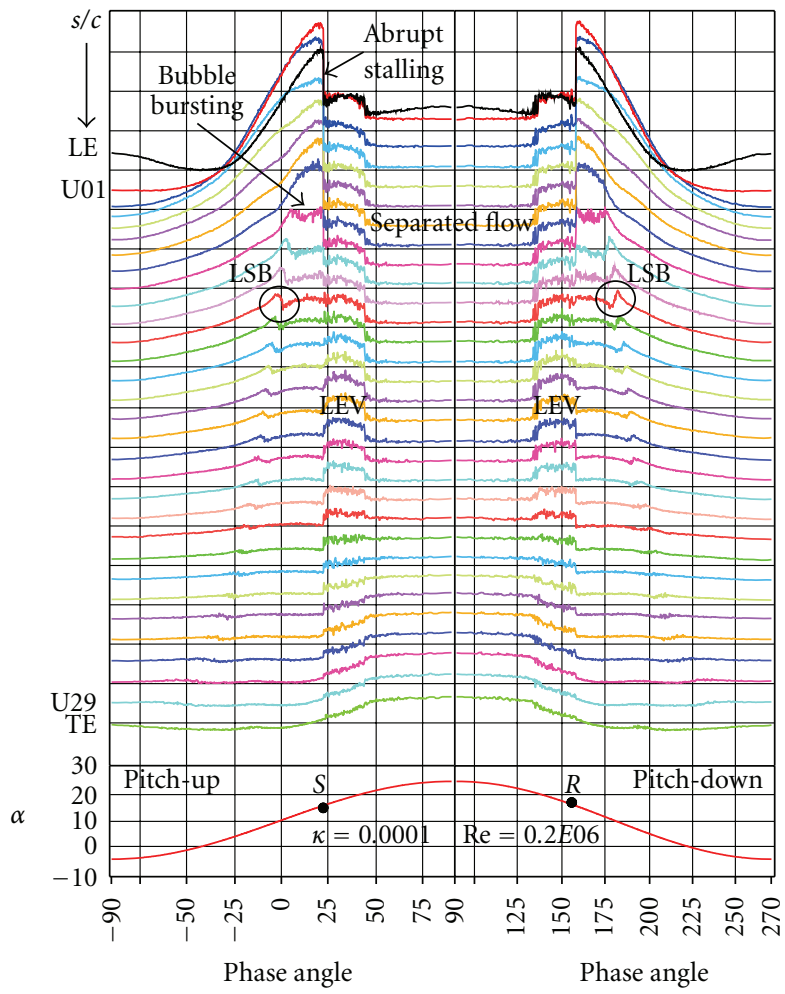

(a)

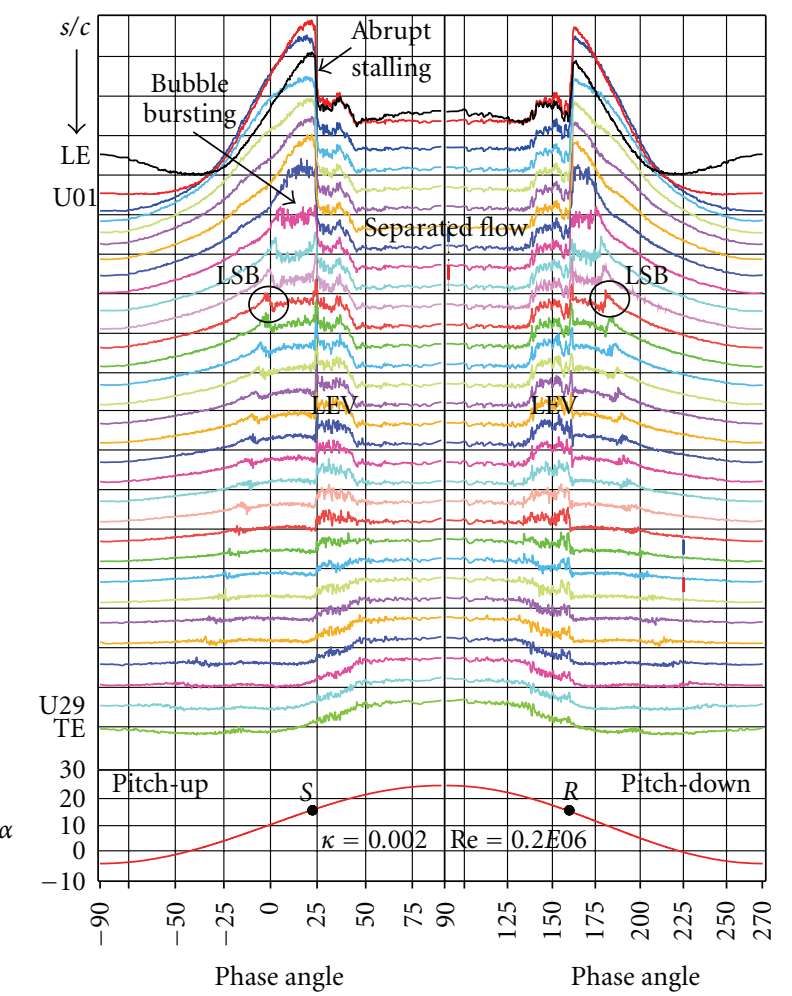

(b)

Figure 11: Time series $C_{P u}$ distribution for $\kappa=0.0001$ and 0.002 at $\operatorname{Re}=0.2 E 06$. 


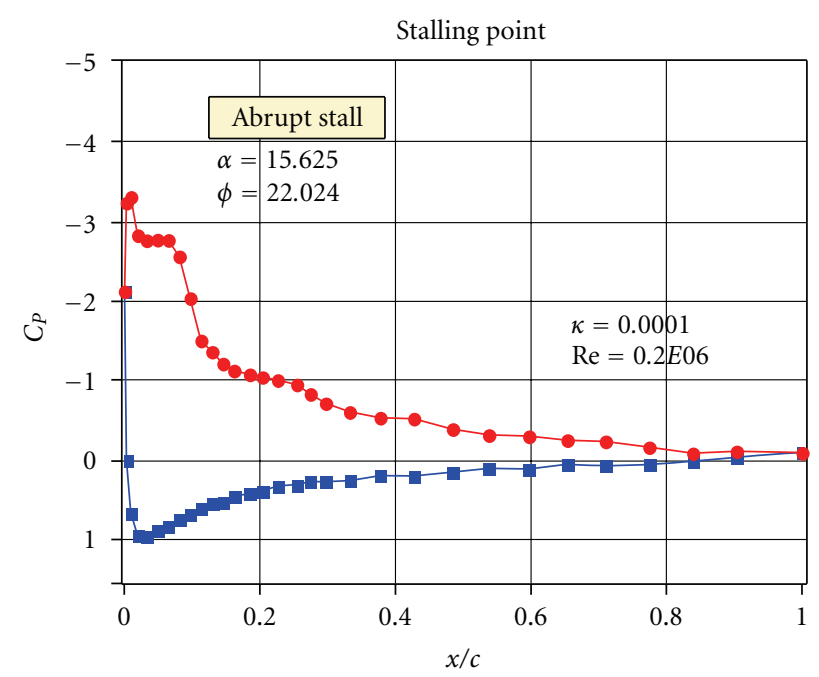

(a)

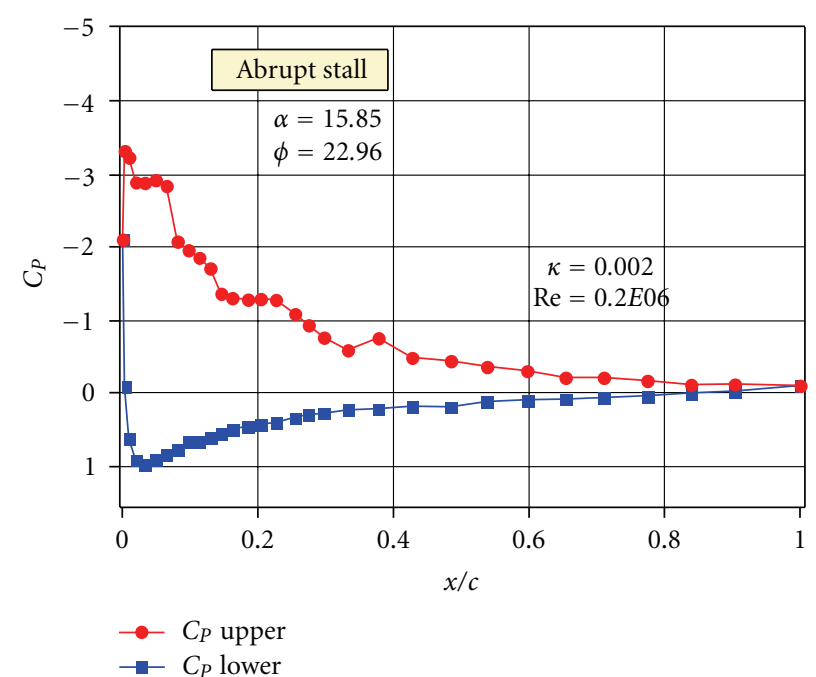

(c)

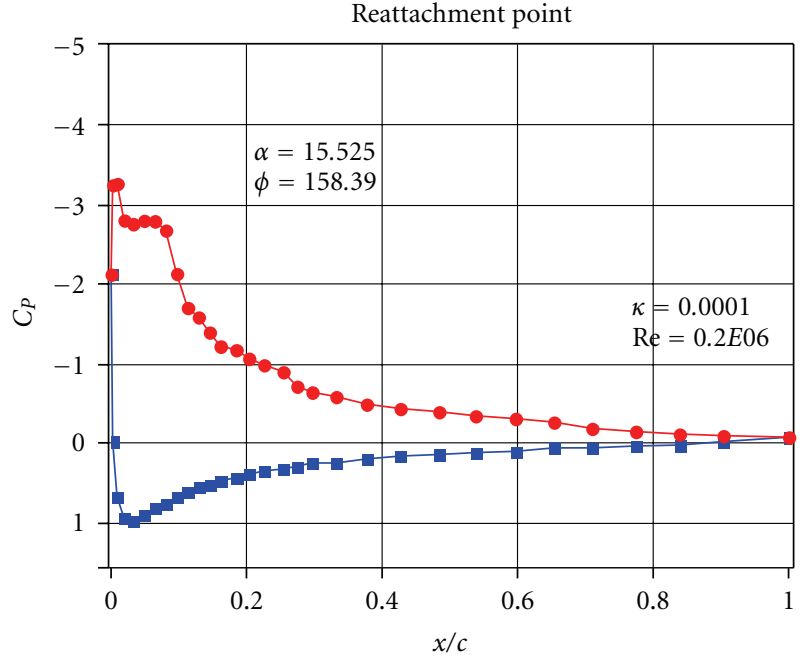

(b)

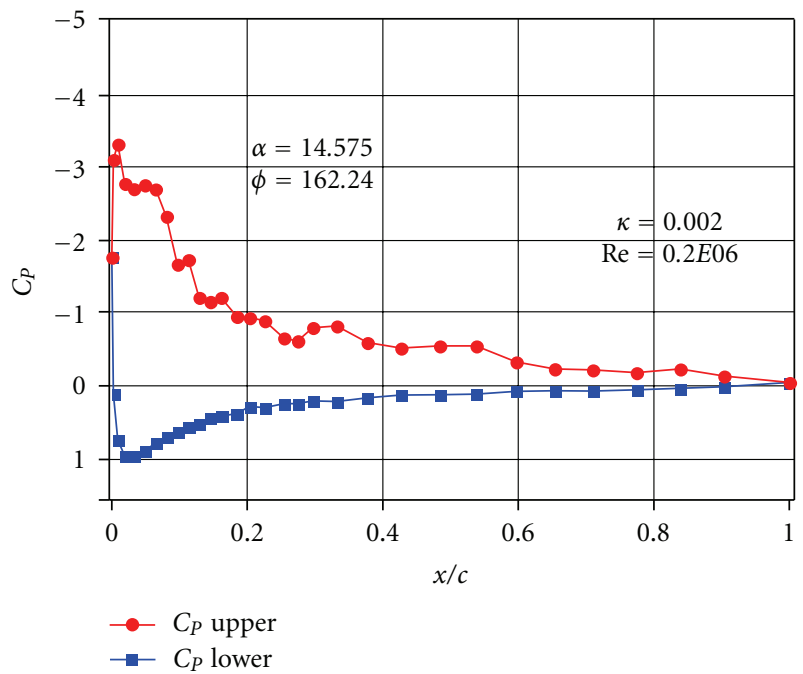

(d)

FIGURE 12: $C_{P}$ Distribution plot at stalling and reattachment points for $\kappa=0.0001$ and 0.002 at constant $\operatorname{Re}=0.2 E 06$.

method adapted for the boundary layer solution of the Navier-Stokes equation [23].

Figure 9 represents the proportionate schematic diagram of the mean flow structure with the evidence of LSB and its distinct features. Location of the boundary layer events shown in \% of chord is measured along the surface starting from the stagnation point.

Figure 10 shows the 3D plot for the standard deviations in $C_{P}$-Signals of the upper surface of the airfoil for $\alpha=-5^{\circ}$ to $25^{\circ}$ at $\operatorname{Re}=0.2 E 06$. With the increase in angle of attack, the growth of transition peak and hence the LSB are clearly depicted from these plots.

\section{Investigation and Determination of Quasi-Steady State}

The steady state analysis does identify and quantify the zone of LSB existing for the given flow configurations. The flow separation is classified under Trailing edge flow separation with evidence of LSB on upstream. Further assessments are carried out by varying $\kappa$ and Re, and quasi-steady flow conditions are critically investigated and determined.

\section{Effect of $\mathcal{\kappa}$ at Constant $\operatorname{Re}$}

The lowest possible reduced frequency of $\kappa=0.0001$ is opted for a given $\mathrm{Re}=0.2 E 06$. Further, step by step, sequentially all the higher values of $\kappa$ are critically analyzed until the frame of time-series $C_{P}$ distribution shows considerable change when compared with $\kappa=0.0001$. It has been found that the time-series $C_{P}$ distribution up to $\kappa=0.002$ shows closure similarity with $\kappa=0.0001$, thus taken for further analysis to determine the limit of quasi-steady state for a given Re. Figure 11 shows the time-series $C_{P u}$ distribution for $\kappa=$ 0.0001 and 0.002 . The symmetric trend in $C_{P}$ plots of pitchup and pitch-down assures the resemblance of flow to the steady state for both the cases. 


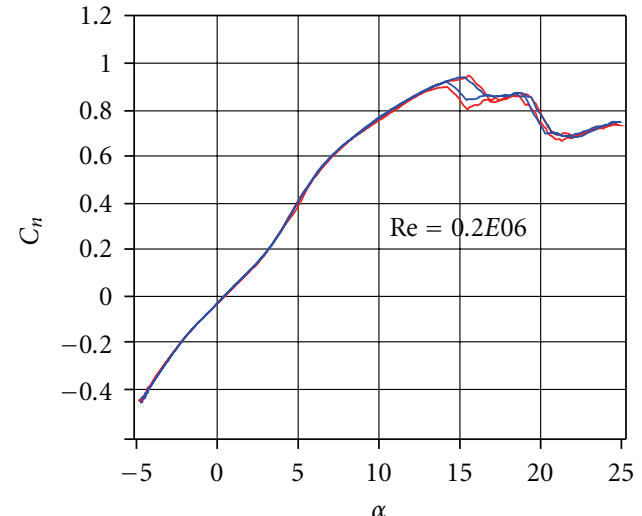

(a)

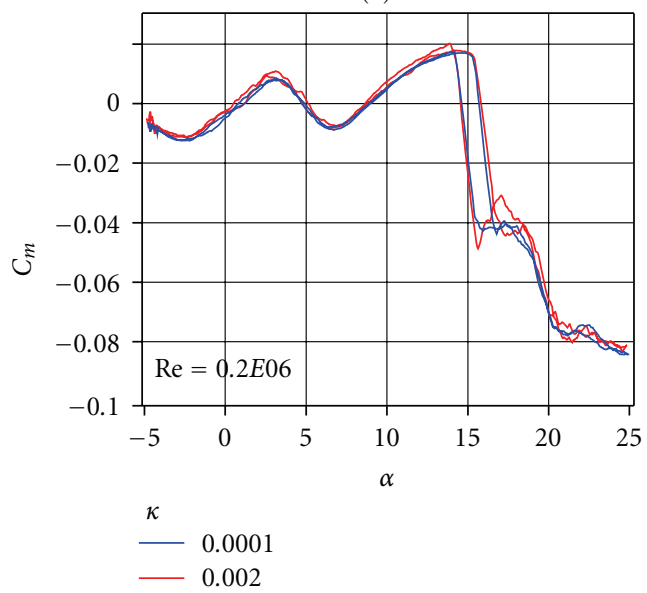

(b)

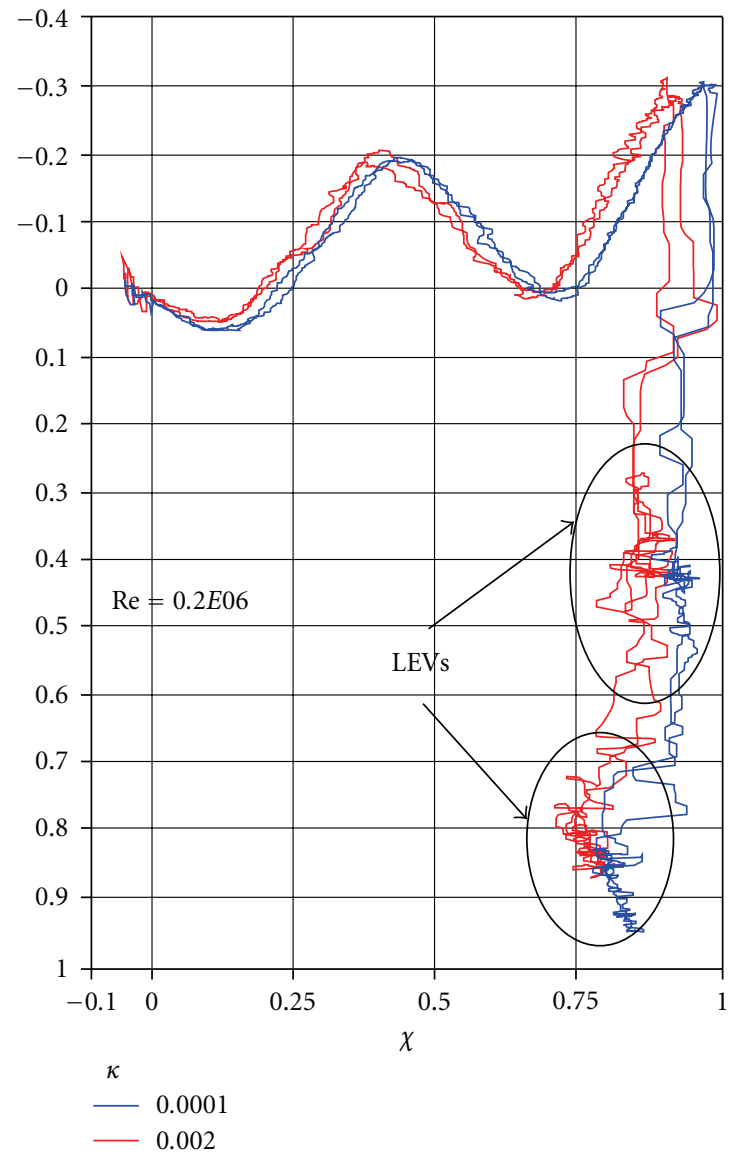

(c)

Figure 13: (a) $C_{n}$ versus $\alpha$, (b) $C_{m}$ versus $\alpha$, (c) $x-\gamma$ plot; for $\kappa=0.0001$ and 0.002 at constant $\operatorname{Re}=0.2 E 06$.

For $\kappa=0.0001$, the strength, onset, and extent of LSB in pitch-up motion are retained in pitch-down motion as well without any phase lag. Similar trends are also observed for $\kappa=0.002$. Effect of reduced frequency change is negligible on LSB characteristics. The suction peak, stalling point, the separation zone, and the reattachment point by large appear identical but need to be closely looked into for more discriminations.

Once the airfoil is stalled, large disturbed wakes are formed since the movement of LEVs continued for a definite phase period $\left(\Delta \phi \approx 23.3^{\circ}\right.$ and $21.18^{\circ}$ for $\kappa=0.0001$ and 0.002 , resp.). However, as evident from the time-series $C_{P u}$ plot, once the LEVs completely shed from the surface, the mean flow near the surface becomes virtually quiescent, leaving only random fluctuations due to local turbulence. Large wake is formed, and thereafter the flow remains fully separated up to $\Delta \phi \approx 88.32^{\circ}$ and $91.25^{\circ}$ for $\kappa=0.0001$ and 0.002 , respectively. During pitch-down motion, LEVs are reintroduced prior to reattachment and continued for considerable phase period $\left(\Delta \phi \approx 25.29^{\circ}\right.$ and $26.03^{\circ}$ for $\kappa=0.0001$ and 0.002 , resp.). The stalling and reattachment points are identified for both the cases, and their chordwise $C_{P}$ distribution plots are shown in Figure 12. For $\kappa=$ 0.0001 negligible phase lag is observed between stalling and reattachment points $\left(\Delta \phi \approx 0.414^{\circ}\right)$ but for $\kappa=0.002$ incremental phase shift is observed. The onset of stalling is delayed by $\Delta \phi_{s} \approx 0.94^{\circ}$, and reattachment point is delayed by $\Delta \phi_{R} \approx 3.85^{\circ}$ to sum up total phage lag of $\Delta \phi \approx 4.79^{\circ}$ for the given change in $\kappa$. No change in suction peak $\left(C_{P} \approx-3.4\right)$ is observed at stalling and reattachment points for both the cases of $\kappa$. The effect of reduced frequency is also visible to cause incremental rise in the fluctuations of pressure waves due to the inception, movement, and shedding of LEVs during pitch-up and pitchdown motion. The fluctuation pattern of $C_{P u}$ for $\kappa=0.002$ as shown in Figures 12(c) and 12(d) indicates intensity and the movement of LEV towards downstream. This is also evident from the $C_{n}-C_{m}$ cross-plots as shown in Figure 13(c) where the progressive movement of cloud of repeating loops indicates the presence, strength, and movement of LEVs. The strength of LEV shows incremental rise with increase in $\kappa$. The $C_{n}$ and $C_{m}$ plots as shown in Figures 13(a) and 13(b) are almost identical for both reduced frequencies. It shows minor hysteresis effect due to minimal phase lag associated with stall behavior as discussed earlier. Also this part of the stall loop is insensitive to the rate of change of angle of attack $\dot{\alpha}$ and remains even when $\dot{\alpha}$ tends to zero, for $\kappa=0.002$, normal force defect $\xi=2.27 \%$, and pitch damping factor $\zeta=-0.0031$. Thus stall flutter of very low amplitude and frequency is incepted and is prolonged due to very low $\dot{\alpha}$ until 


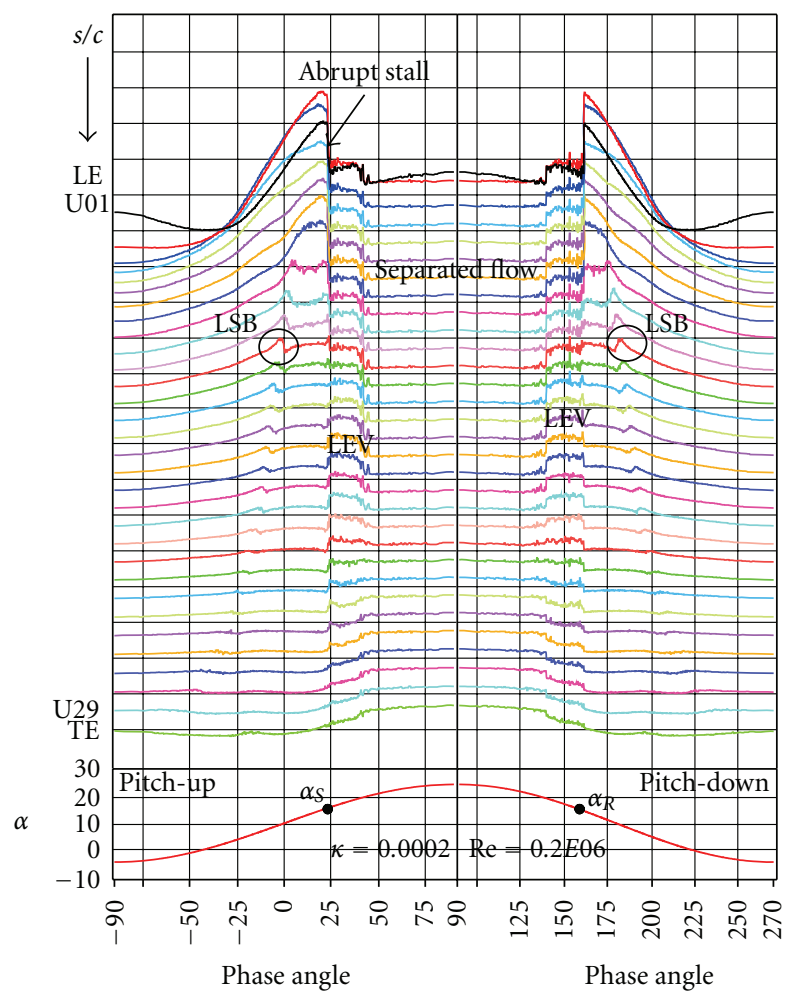

(a)

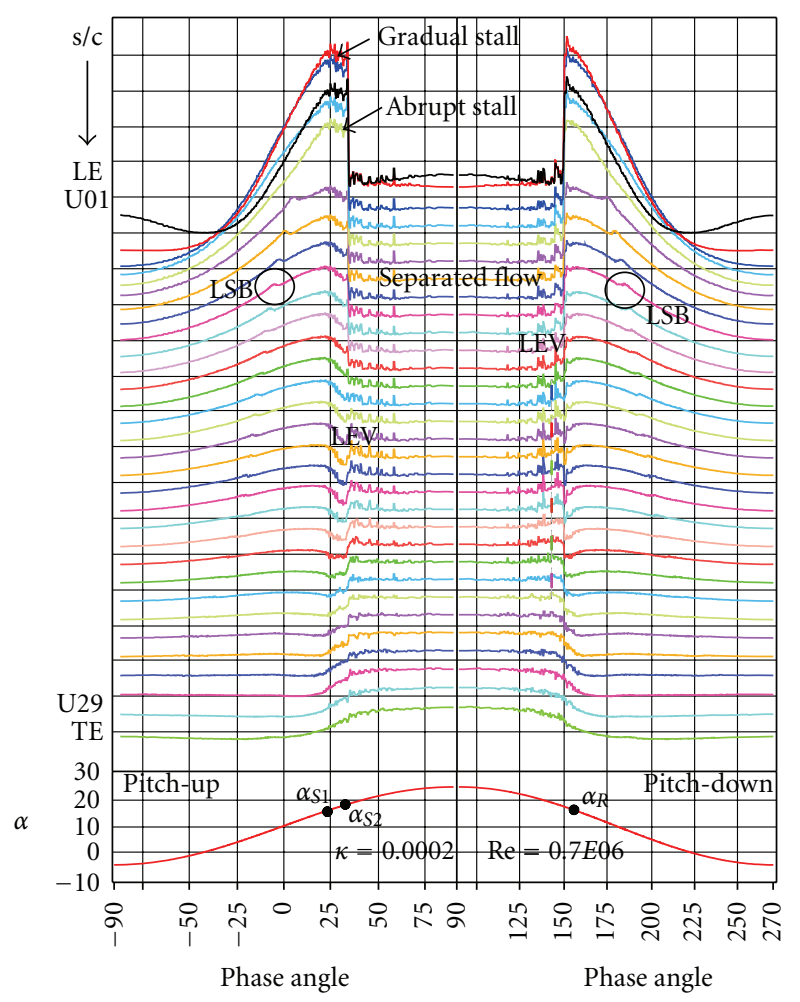

(b)

Figure 14: Time series $C_{P u}$ distribution for $\kappa=0.002$ at $\operatorname{Re}=0.2 E 06$ and 0.7E06.

it get restrained and hence promotes the minor quasi-steady hysteresis. The quantum of varied phase lags, strength of LEVs, and stall flutter associated with the change in $\kappa$ is very low as compared to larger domain of unsteady characteristics and hence does not seriously affect the stall characteristics and are widely comparable to its steady counterpart. Thus $\kappa=0.002$ could be considered as the limiting condition for defining quasi-steady state at $\operatorname{Re}=0.2 E 06$.

Similarly, limiting reduce frequencies for quasi-steady state are identified and validated for all the selected range of Re and are summarized in Figure 17. Effect of Re for a given reduced frequency in the domain of quasi-steady state is assessed further.

\section{Effect of Re at Constant $\kappa$}

The effect of Re of dominant and obvious in quasi-steady state, and the characteristics attained are comparable to its baseline characteristics at varied Re. A very low reduced frequency, $\kappa=0.0002$, is opted for analysis which lies well within the limiting value defining the quasi-steady state for the given range of Re. Here the two extreme Re are taken for comparison, which through baseline tests are categorized under different stall regimes. As shown in Figure 14, $\operatorname{Re}=0.2 E 06$ undergoes abrupt stalling $\left(\alpha_{S}\right)$ during pitchup motion, and also its reattachment $\left(\alpha_{R}\right)$ is instantaneous during pitch-down motion. While, for $\mathrm{Re}=0.7 E 06$, gradual stall $\left(\alpha_{S 1}\right)$ followed by abrupt stall $\left(\alpha_{S 2}\right)$, referred as double stall, is evident during pitch-up motion but during pitchdown motion, the reattachment $\left(\alpha_{R}\right)$ is instantaneous. For a given Re, the strength, onset, and extent of LSB in pitch-up motion are retained in pitch-down motion as well without any time lag but an increase in Re causes significant changes in the characteristics of LSB. With the increase in Re, the onset of LSB and transition path is confined but the strength of LSB considerably reduces and LSB gets extended further upstream closure to the vicinity of LE. For Re $=0.2 E 06$, LSB has moved up to port ID-U08 $(s / c=11.88 \%$ of chord measured from the LE) and got stuck there to trigger bubble bursting followed by abrupt stalling. For Re $=0.7 E 06$, the weak LSB is evident up to port ID- U05 $(s / c=6.84 \%)$. At this location LSB bursts but the energy impact due to bursting is considerably low as compared to the entrainment energy from the free stream thus results in the extension of TBL and drop in $C_{P}$, promoting gradual stall. Later on, due to further rise in $\alpha$, TBL separates to promote abrupt stalling.

Here one thing is significantly noted that during pitchup motion, in abrupt stall case, LEV is incepted after abrupt stalling and prolongs for a definite phase period $(\Delta \phi \approx$ $19.77^{\circ}$ ) followed by a separation zone of $\Delta \phi \approx 96.39^{\circ}$ and reintroduction of LEV for a phase period of $\Delta \phi \approx$ $22.31^{\circ}$ prior to reattachment. But in double stall case, LEV is incepted during gradual stalling, and weak evidence of LEV is tracked, for a phase period of $\Delta \phi \approx 24.89^{\circ}$, once the airfoil is abruptly stalled followed by a full separation zone of $\Delta \phi \approx 66.24^{\circ}$. Weak LEVs are reintroduced for a phase period of $\Delta \phi \approx 25.29^{\circ}$ prior to reattachment. Thus 


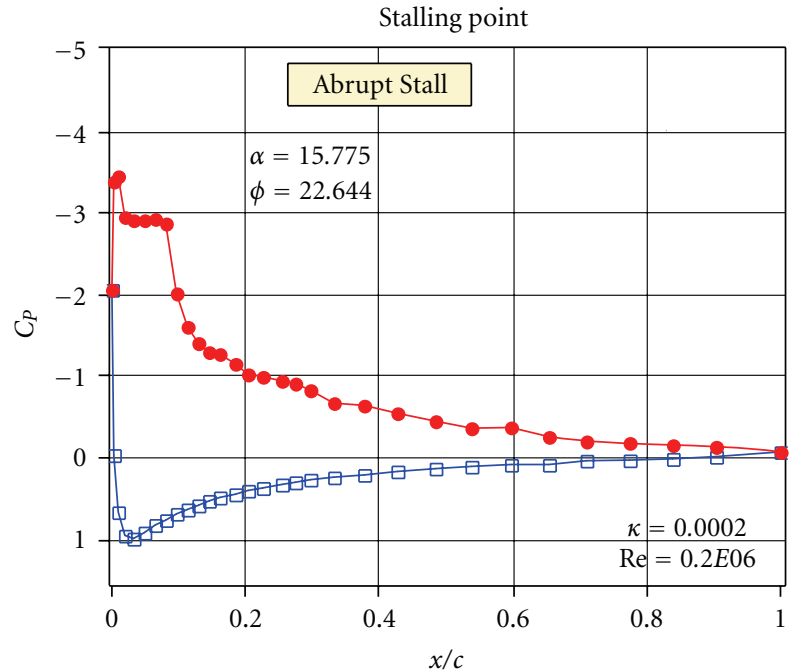

(a)

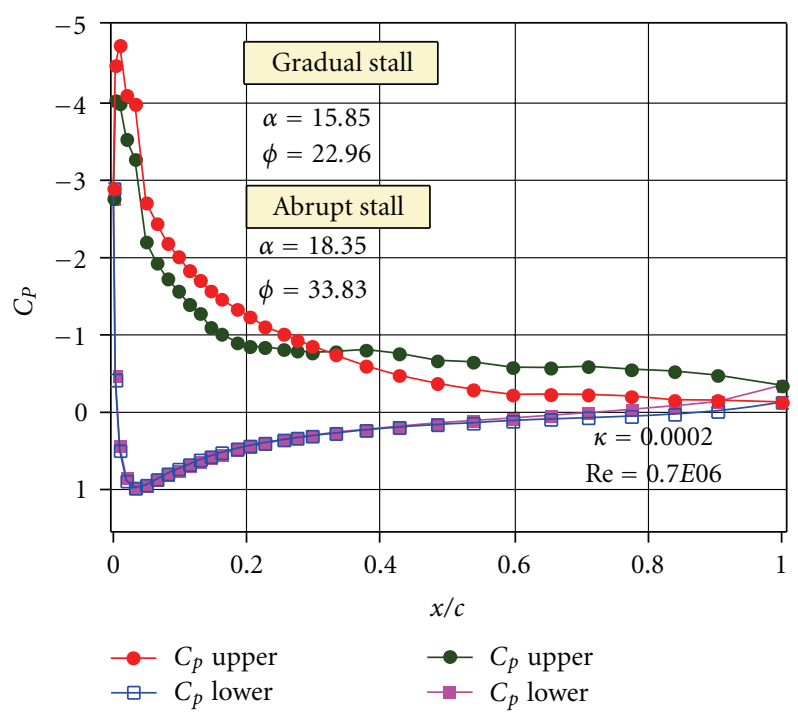

(c)

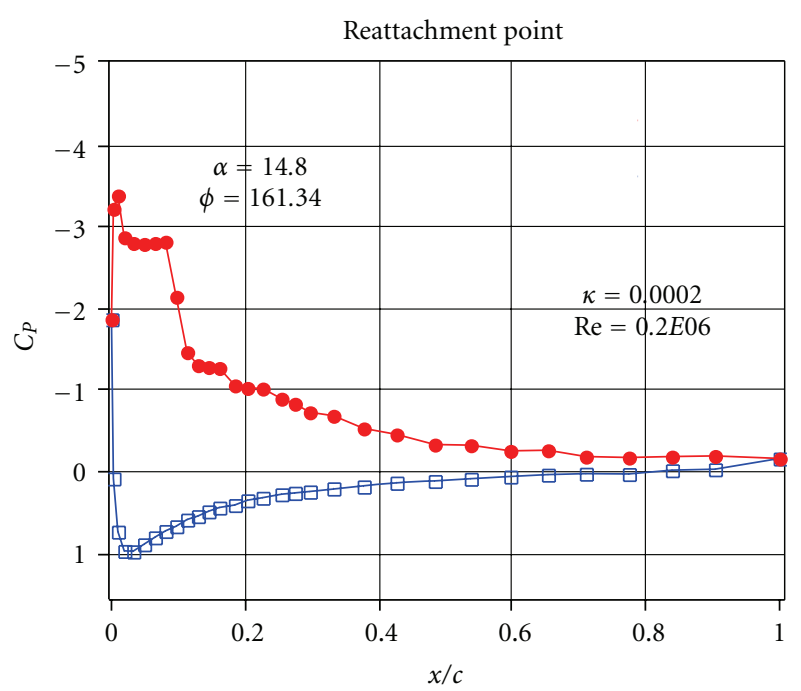

(b)

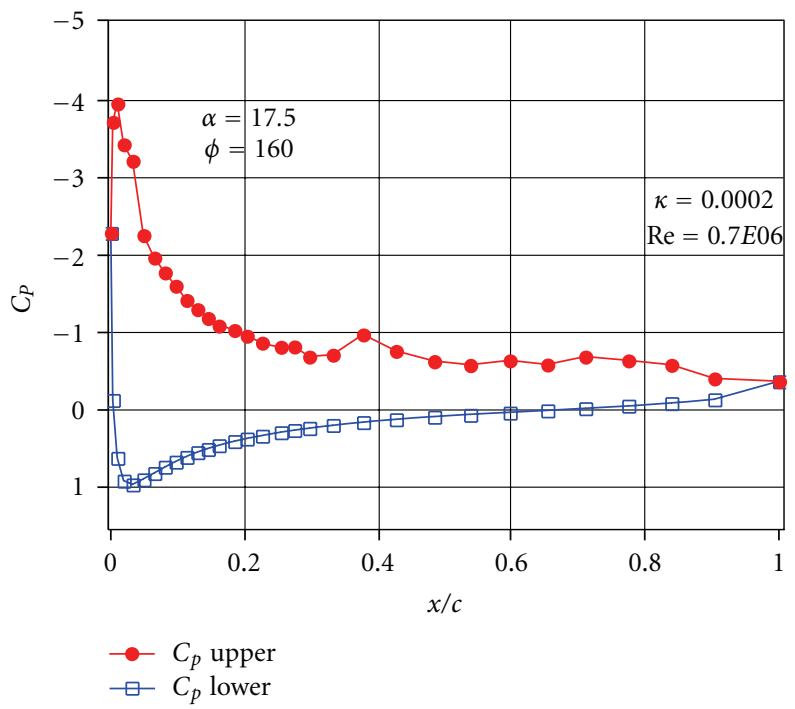

(d)

FIgURE 15: $C_{p}$ Distribution plot at stalling and reattachment points for $\operatorname{Re}=0.2 E 06$ and $0.7 E 06$ at constant $\kappa=0.0002$.

increase in Re shows substantial reduction in the intensity of LEV without any considerable change in the phase. This can also be observed in the $C_{n}-C_{m}$ plots shown in Figure 16(c). Figure 15 shows the chord-wise $C_{P}$ distribution plots at the onset of stalling and reattachment points for both the opted Re cases at $\kappa=0.0002$. As shown in Figures 15(a) and 15(b), the phase lag between the stalling and reattachment for $\operatorname{Re}=0.2 E 06$ is $\Delta \phi=\phi_{S}-\phi_{R} \approx 3.98^{\circ}$ with no change in its suction peak $\left(C_{P} \approx-3.4\right)$. For the double stall case at $\operatorname{Re}=0.7 E 06$, as shown in Figures 15(c) and 15(d), the phase lag between the inceptions of gradual and abrupt stall is $\Delta \phi=\phi_{S 2}-\phi_{S 1} \approx 10.97^{\circ}$. The reattachment $C_{P}$-plot is found to be more comparable to the onset of abrupt stall $C_{P}$ plot with no evitable change in suction peak $\left(C_{P} \approx-4\right)$. Also the phase lag between the two is found to be significant $\Delta \phi=$ $\phi_{S 2}-\phi_{R} \approx 13.83^{\circ}$, but when compared to the onset of gradual stall the phase lag is reduced to $\Delta \phi=\phi_{S 1}-\phi_{R} \approx 2.96^{\circ}$ with a drop in suction peak as well from $C_{P} \approx-4.8$ to $C_{P} \approx$ -4 . Thus, individually for each Re cases, there is not any considerable phase lag between the onset of stalling and reattachment point. Figure 16(a) shows incremental rise in $C_{n}$ are reintroduced for a phase period of $\Delta \phi \approx 25.29^{\circ}$ prior to reattachment. Thus increase in Re shows substantial reduction in the intensity of LEV without any considerable change in the phase. This can also be observed in the $C_{n}-$ $C_{m}$ plots are shown in Figure 16(c). Figure 15 shows the chord-wise $C_{P}$ distribution plots at the onset of stalling and reattachment points for both opted Re cases at $\kappa=0.0002$. As shown in Figures 15(a) and 15(b), the phase lag between the stalling and reattachment for $\operatorname{Re}=0.2 E 06$ is $\Delta \phi=$ $\phi_{S}-\phi_{R} \approx 3.98^{\circ}$ with no change in its suction peak $\left(C_{P} \approx\right.$ -3.4 ). For the double stall case at $\mathrm{Re}=0.7 E 06$, as shown in Figures $15(\mathrm{c})$ and $15(\mathrm{~d})$, the phase lag between the inceptions of gradual and abrupt stall is $\Delta \phi=\phi_{S 2}-\phi_{S 1} \approx 10.97^{\circ}$. The 


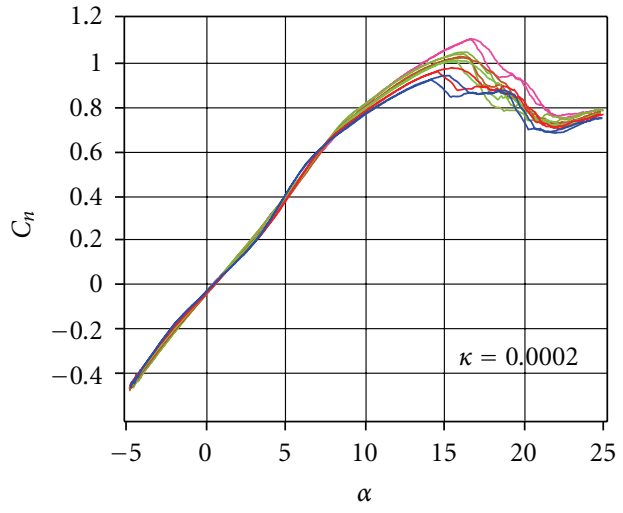

(a)

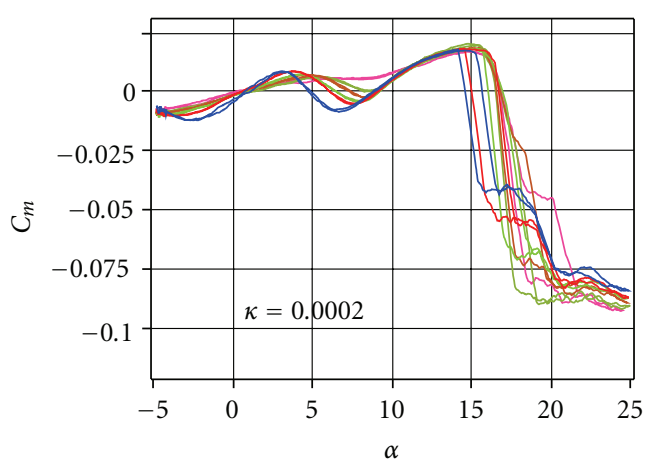

(b)

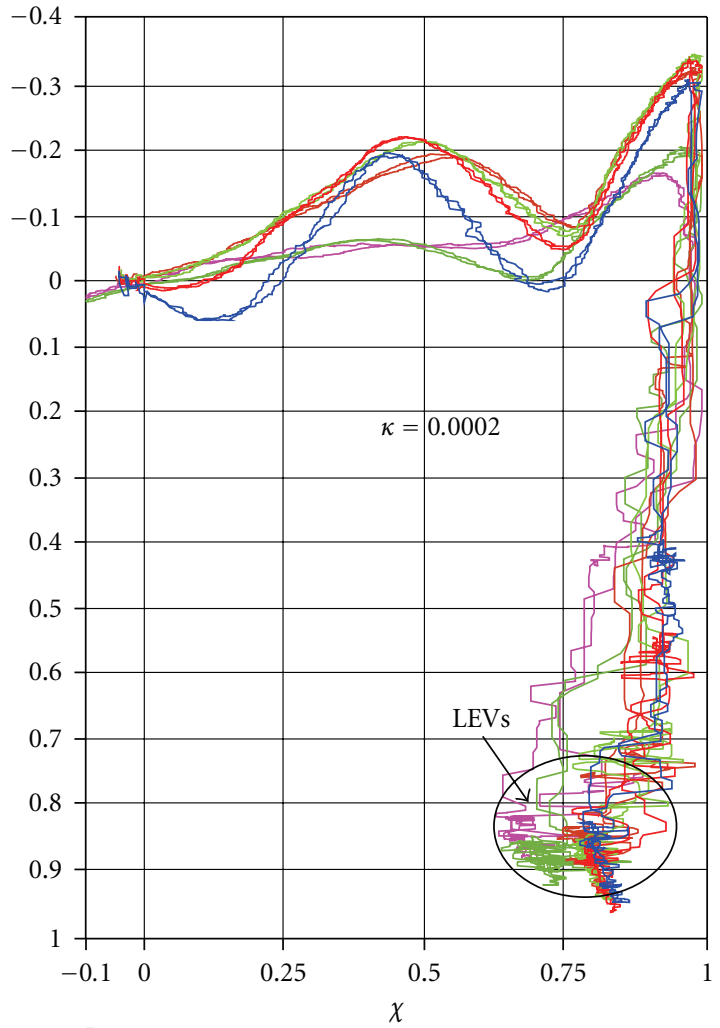

$\operatorname{Re}$

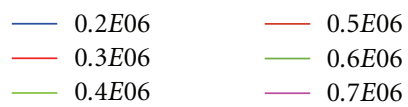

(c)

Figure 16: (a) $C_{n}$ versus $\alpha$, (b) $C_{m}$ versus $\alpha$, (c) $x-\gamma$ cross plot; at $\kappa=0.0002$ for varied $\operatorname{Re}=0.2 E 06$ to $0.7 E 06$.

reattachment $C_{P}$-plot is found to be more comparable to the onset of abrupt stall $C_{P}$-plot with no evitable change in suction peak $\left(C_{P} \approx-4\right)$. Also the phase lag between the two is found to be significant $\Delta \phi=\phi_{S 2}-\phi_{R} \approx 13.83^{\circ}$, but when compared to the onset of gradual stall the phase lag is reduced to $\Delta \phi=\phi_{S 1}-\phi_{R} \approx 2.96^{\circ}$ with a drop in suction peak as well from $C_{P} \approx-4.8$ to $C_{P} \approx-4$. Thus, individually for each Re case, there is not any considerable phase lag between the onset of stalling and reattachment point. Figure 16(a) shows incremental rise in $C_{n}$ and stalling angle with the increase in Re. The minor hysteresis observed at lower Re gets reduced with the increase in Re. As shown in Figures 16(b) and $16(\mathrm{c})$, the peaks and valleys formed in $C_{m}$-plots and $C_{n}-C_{m}$ plots in the prestall region indicate the dominance of LSB which further becomes flatter and flatter with the rise in Re, reducing the impact of LSB and promoting the extent of turbulent boundary layer separation. Thus, by increasing the Re beyond $0.7 E 06$ to a certain critical value, onset of LSB could get completely extinct from the flow phenomena. The extent of separated flow (full stall conditions) reduces with the increase in Re thus transforms large wake at lower Re to comparatively smaller wake.

The normal force defect and pitch damping factor calculated for each case of varied Re and $\kappa$ within the quasisteady domain are plotted and summarized in Figure 17.

\section{Conclusions}

The quasi-steady conditions for the varied range of Re and $\kappa$ are determined and summarized in Figure 17.

The limiting value of reduced frequency for quasi-steady state decreases with the increase in Re. The effect of Re is dominant for a given $\kappa$ but the effect of $\kappa$ for a given Re is negligible. However for any Re and $\kappa$ within the quasisteady domain, the phase lag interpreted with the onset of stall and reattachment is very low and comparable to its steady counterparts. Also the onset of LEVs, weather weak for strong, is incepted after stalling promotes boundary layer separation followed by the formation of large wake. Thus the boundary layer separation and reattachment is analogous to steady flow conditions.

Normal force defect $\xi \leq 2.27 \%$ and Pitch damping factor $\zeta<0$ hint at instability in the oscillating motion with the inception of stall flutter. This may form minor quasi-steady hysteresis which reduces with the increase in Re. The flow condition of pitch-up motion by and large gets retained during the pitch-down motion without any considerable affect on the stall characteristics comparable to its steady counterpart.

The onset and extent of LSB, if evident for the given range of Re, are unaffected by any change in $\kappa$. Effect of Re does 

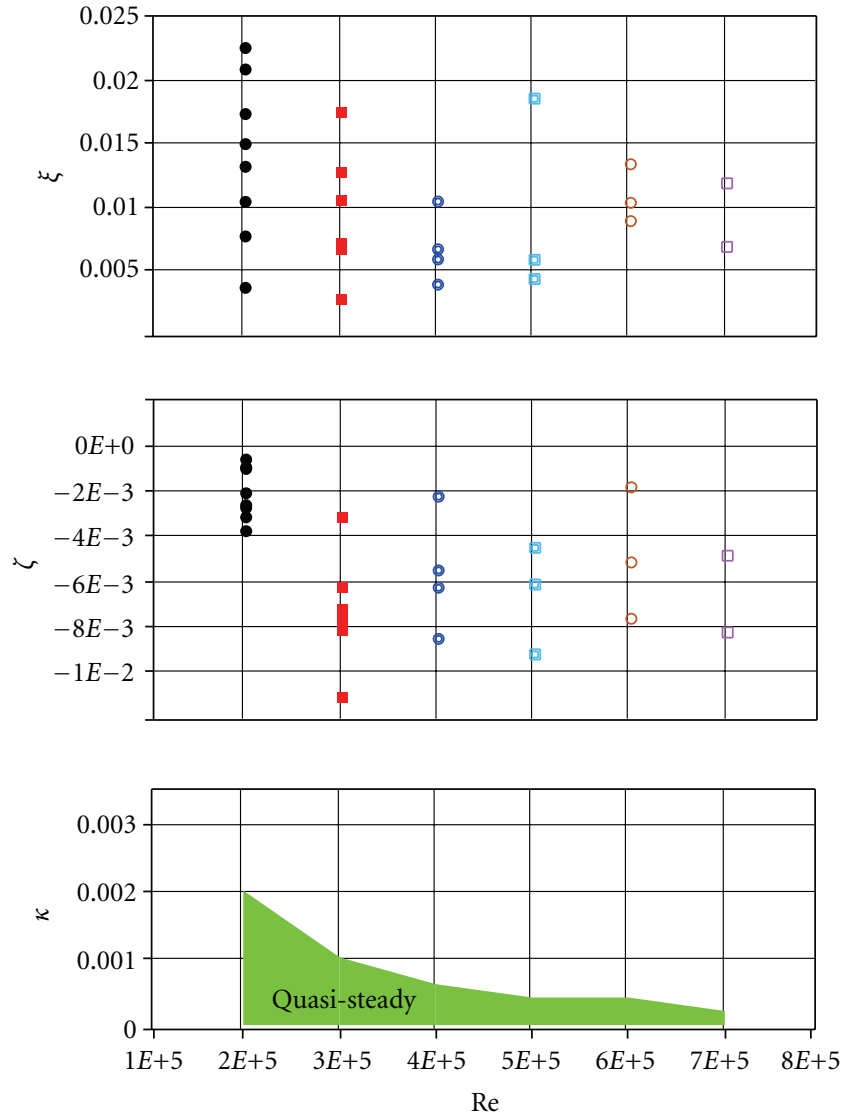

FIGURE 17: Summary of results obtained from quasi-steady tests.

reduce the strength of LSB and increases its extent toward upstream without any change in its onset phase. In short, the transition path is conserved for any change in Re and $\kappa$. This impact of LSB and the transition path has been assessed in the quasi-steady domain but needs to be critically assessed further for unsteady flow conditions.

\section{Acknowledgments}

The present paper is financially supported by the Aeronautics and Research Development Board (ARDB) of India. Technical support and assistance provided by the staff of Low speed aerodynamics lab and National Wind Tunnel Facility (NWTF) at IIT-Kanpur are gratefully acknowledged.

\section{References}

[1] W. Johnson and N. D. Ham, "On the mechanism of dynamic stall," Journal of American Helicopter Society, vol. 17, no. 4, pp. 36-45, 1972.

[2] J. M. Martin, R. W. Empey, W. J. McCroskey, and F. K. Caradonna, "An experimental analysis of dynamic stall on an oscillating airfoil," Journal of the American Helicopter Society, vol. 19, no. 1, pp. 26-32, 1974.

[3] W. J. McCroskey and J. J. Philippe, "Unsteady viscous flow on oscillating airfoils," AIAA Journal, vol. 13, no. 1, pp. 71-79, 1975.
[4] W. J. McCroskey, K. W. McAlister, L. W. Carr, S. L. Pucci, O. Lambert, and R. F. Indergrand, "Dynamic stall on advanced airfoil sections," Journal of the American Helicopter Society, vol. 26, no. 3, pp. 40-50, 1981.

[5] L. W. Carr, "Progress in analysis and prediction of dynamic stall," Journal of Aircraft, vol. 25, no. 1, pp. 6-17, 1988.

[6] L. E. Ericsson and J. P. Reding, "Dynamic overshoot of static stall angle," Journal of Aircraft, vol. 22, no. 7, pp. 637-638, 1985.

[7] L. E. Ericsson, "Moving wall effects in unsteady flow," Journal of Aircraft, vol. 25, no. 11, pp. 977-990, 1988.

[8] J. M. Walker, H. E. Helin, and J. H. Strickland, "An experimental investigation of an airfoil undergoing large-amplitude pitching motions," AIAA Journal, vol. 23, no. 8, pp. 1141-1142, 1985.

[9] K. Biber and G. W. Zumwalt, "Hysteresis effects on wind tunnel measurements of a two-element airfoil," AIAA Journal, vol. 31, no. 2, pp. 326-330, 1993.

[10] R. B. Green and R. A. M. Galbraith, "Phenomena observed during aerofoil ramp-down motions from the fully separated state," Aeronautical Journal, vol. 98, no. 979, pp. 349-356, 1994.

[11] C. Shih, L. M. Lourenco, and A. Krothapalli, "Investigation of flow at leading and trailing edges of pitching-up airfoil," AIAA Journal, vol. 33, no. 8, pp. 1369-1376, 1995.

[12] M. J. Rhee, "A study of dynamic stall vortex development using two-dimensional data from the AFDD oscillating wing experiment," Tech. Rep. NASA/TM-2002-211857, AFDD/TR02-A-009, 2002.

[13] S. Mittal and P. Saxena, "Hysteresis in flow past a NACA 0012 airfoil," Computer Methods in Applied Mechanics and Engineering, vol. 191, no. 19-20, pp. 2179-2189, 2002.

[14] A. Ferrecchla, F. N. Coton, R. A. M. Galbralth, and R. B. Green, "An investigation of dynamic stall onset on a pitching wing," Aeronautical Journal, vol. 107, no. 1074, pp. 487-494, 2003.

[15] M. R. Soltani and A. R. Davari, "On self-similar behaviour of the hysteresis loops in pitching motions," Aeronautical Journal, vol. 108, no. 1086, pp. 427-434, 2004.

[16] W. J. McCroskey, "Unsteady airfoils," Annual Review of Fluid Mechanics, vol. 14, pp. 285-311, 1982.

[17] L. W. Carr, K. W. McAlister, and W. J. McCroskey, "Analysis of the development of dynamic stall based on oscillating airfoil experiments," Tech. Rep. NASA TN D-8382, 1977.

[18] S. Goldstein, "On laminar boundary-layer flow near a position of separation," Quarterly Journal of Mechanics and Applied Mathematics, vol. 1, no. 1, pp. 43-69, 1948.

[19] W. R. Sears and D. P. Telionis, "Boundary-layer separation in Unsteady Flow," SIAM Journal on Applied Mathematics, vol. 28, no. 1, pp. 215-235, 1975.

[20] J. F. Nash, L. W. Carr, and R. E. Singleton, "Unsteady turbulent boundary layers in two-dimensional incompressible flow," AIAA Journal, vol. 13, no. 2, pp. 167-173, 1975.

[21] K. W. McAlister, L. W. Carr, and W. J. McCroskey, "Dynamic stall experiments on the NACA 0012 airfoil," Tech. Rep. NASA TP 1100, 1978.

[22] L. E. Ericsson, "Comments on unsteady airfoil stall," Journal of Aircraft, vol. 4, no. 5, pp. 478-480, 1967.

[23] D. M. Sharma and K. Poddar, "Experimental investigations of Laminar separation bubble for a flow past an Airfoil," in Proceedings of the ASME Turbo-Expo Conference, Glasgow, UK, June 2010. 

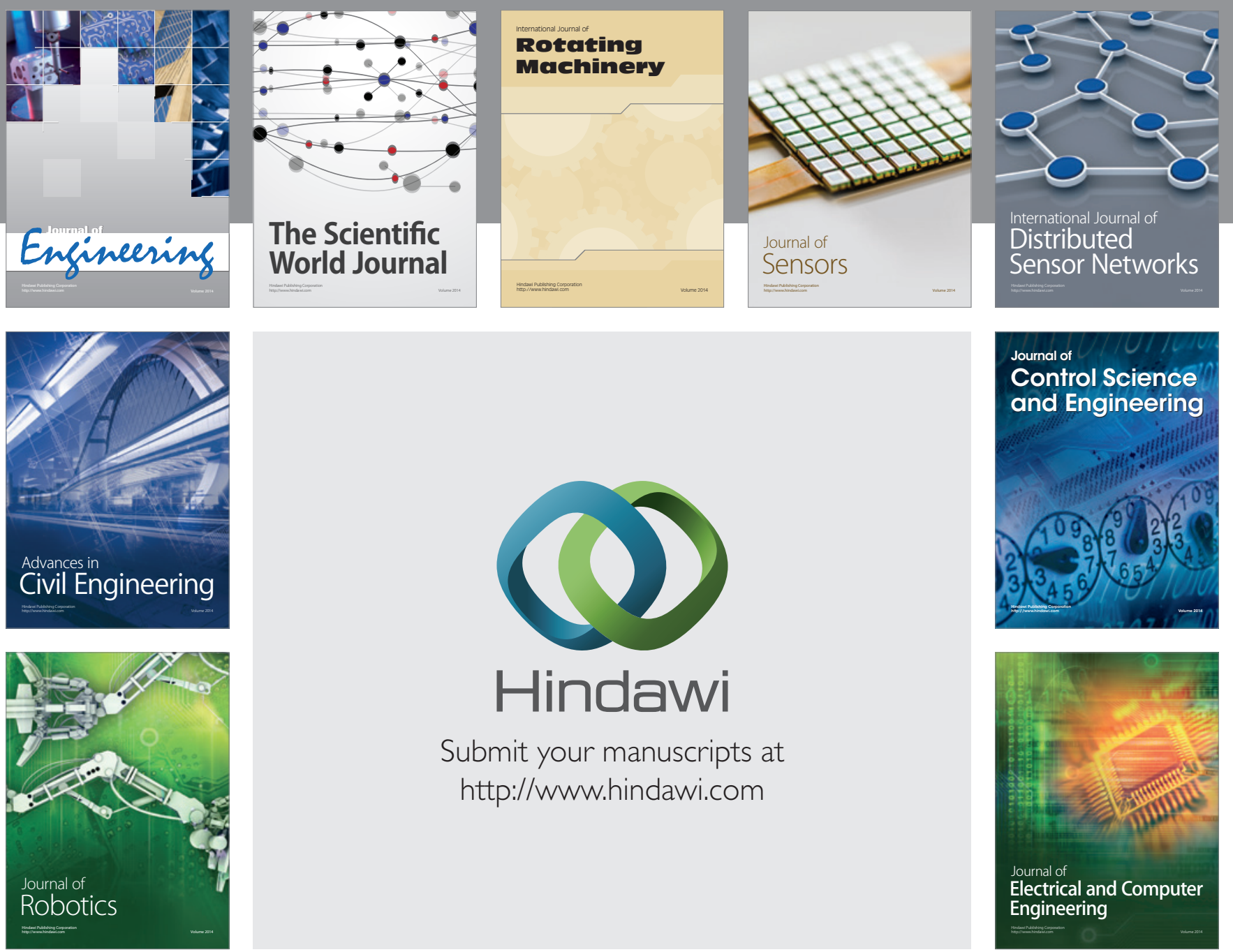

Submit your manuscripts at

http://www.hindawi.com
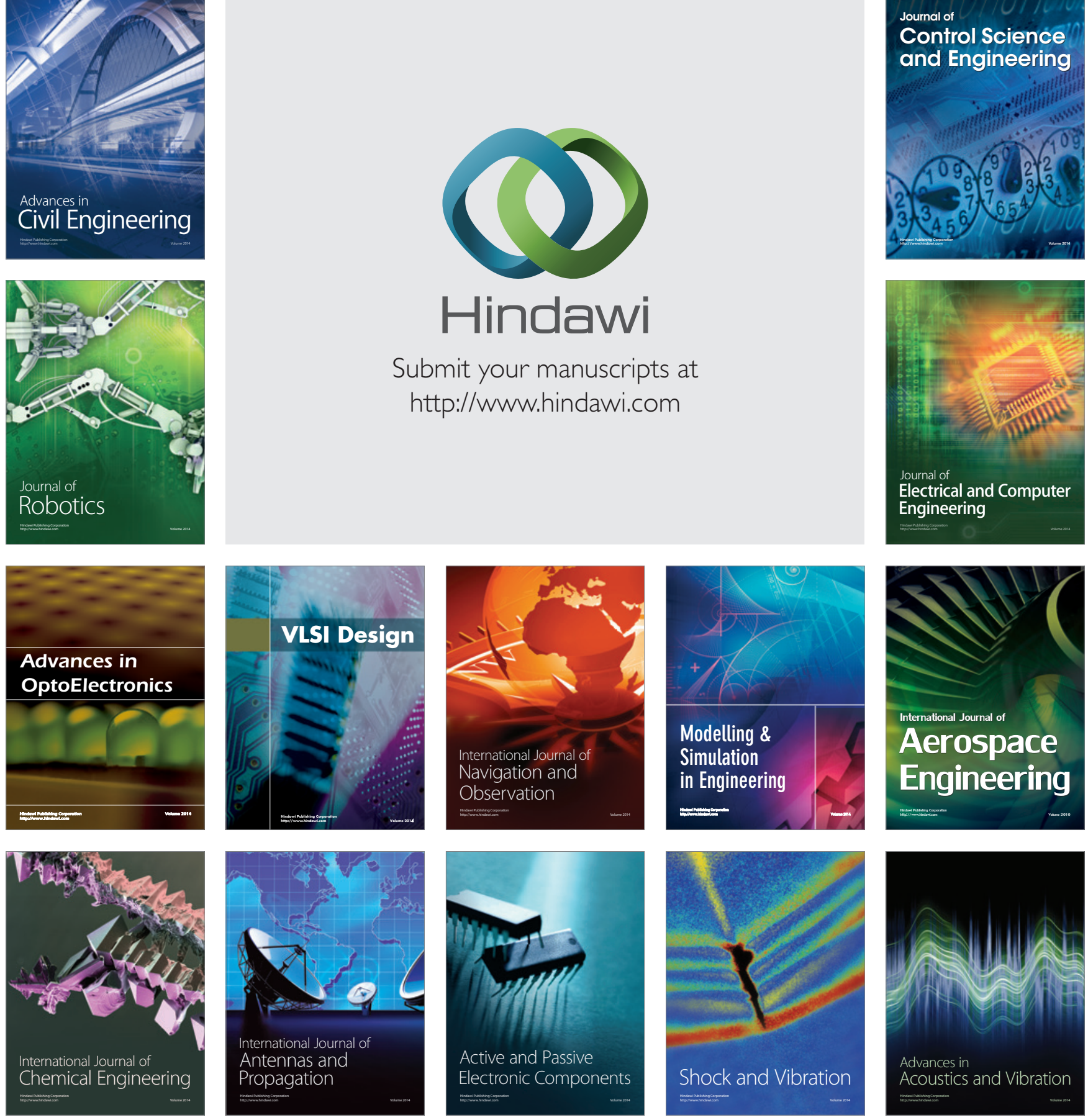